\title{
The Induction of Tomato Leucine Aminopeptidase Genes (LapA) After Pseudomonas syringae pv. tomato Infection Is Primarily a Wound Response Triggered by Coronatine
}

\author{
Véronique Pautot, ${ }^{1}$ Frances M. Holzer, ${ }^{2}$ Josette Chaufaux, ${ }^{3}$ and Linda L. Walling ${ }^{2}$ \\ ${ }^{1}$ Laboratoire de Biologie Cellulaire, Institut National de la Recherche Agronomique, Route de St. Cyr, 78026 \\ Versailles Cédex, France; ${ }^{2}$ Department of Botany and Plant Sciences, University of California, Riverside \\ 92521-0124; '3Station de Recherches de Lutte Biologique, La Minière, 78285 Guyancourt Cédex, Institut \\ National de la Recherche Agronomique, France \\ Accepted 11 October 2000.
}

Tomato plants constitutively express a neutral leucine aminopeptidase (LAP-N) and an acidic LAP (LAP-A) during floral development and in leaves in response to insect infestation, wounding, and Pseudomonas syringae pv. tomato infection. To assess the physiological roles of LAP-A, a LapA-antisense construct (35S:asLapA1) was introduced into tomato. The 35S:asLapA1 plants had greatly reduced or showed undetectable levels of LAP-A and LAP-N proteins in healthy and wounded leaves and during floral development. Despite the loss of these aminopeptidases, no global changes in protein profiles were noted. The 35S:asLapA1 plants also exhibited no significant alteration in floral development and did not impact the growth and development of Manduca sexta and $P$. syringae pv. tomato growth rates during compatible or incompatible infections. To investigate the mechanism underlying the strong induction of LapA upon $P$. syringae pv. tomato infection, LapA expression was monitored after infection with coronatine-producing and -deficient $P$. syringae pv. tomato strains. LapA RNA and activity were detected only with the coronatine-producing $P$. syringae pv. tomato strain. Coronatine treatment of excised shoots caused increases in RNAs for jasmonic acid (JA)-regulated wound-response genes (LapA and pin2) but did not influence expression of a JA-regulated pathogenesis-related protein gene $(P R-1)$. These results indicated that coronatine mimicked the wound response but was insufficient to activate JA-regulated $P R$ genes.

Additional keywords: defense response, exopeptidases, Lycopersicon esculentum, methyl jasmonate, octadecanoid pathway, phytotoxin.

Leucine aminopeptidases (LAPs) (E.C.3.4.11.1) catalyze the cleavage of N-terminal amino acids from peptides and proteins. These metalloproteases are widely distributed in the

Corresponding author: L. L. Walling; Telephone: +1-909-787-4687; Fax:+1-909-787-4437; E-mail: Lwalling@ citrus.ucr.edu plant and animal kingdoms, exhibit a high level of amino acid identity, and have similarities in their substrate specificities (Gu and Walling 2000; Gu et al. 1999; Rawlings and Barrett 1993; Taylor et al. 1996; Walling and Gu 1996). In animals, the bovine lens LAP was one of the first aminopeptidases characterized (Smith and Hill 1960). Despite the number of biochemical and biophysical studies that have accumulated on the animal LAP (Kim and Lipscomb 1994; Taylor et al. 1996), the exact role of LAP remains to be established. The animal LAP may be essential for protein turnover because altered LAP activity has been associated with a variety of pathologies, including protein deposition during cataract formation in the lens (Stuart and Doughty 1996; Taylor et al. 1993). In addition, the human LAP is induced by interferon- $\gamma$ (Harris et al. 1992) and is important for the processing of peptides released by the $26 \mathrm{~S}$ proteasome for presentation to major histocompatibility complex (Beninga et al. 1998).

cDNAs encoding LAP enzymes have been described in Arabidopsis thaliana, potato, and tomato (Bartling and Weiler 1992; Hildmann et al. 1992; Pautot et al. 1993; Walling and $\mathrm{Gu}$ 1996). There are two classes of 55-kDa LAP proteins in tomato that can be distinguished by their pIs and differential patterns of expression ( $\mathrm{Gu}$ et al. 1996b). The neutral LAP-N proteins are expressed constitutively and found in all monocots and dicots examined to date (Chao et al. 2000; Gu et al. 1996b). The tomato LAP-N proteins are most similar to the LAP described in A. thaliana (Bartling and Nosek 1994) and are likely to be similar to the seed LAPs purified from kidney bean and barley (Mikkonen 1992; Sopanen and Mikola 1975).

The acidic LAP-A proteins accumulate in all developing floral organs and, more specifically, in tomato ovary integument, suggesting a nutritional role of LAP-A for the developing embryo (Chao et al. 1999; Milligan and Gasser 1995; Ruíz-Rivero and Prat 1998; C.-J. Tu and L. Walling unpublished results). In addition, LAP-A appears to be part of the plant-defense response because the LapA RNAs and proteins accumulate to high levels in tomato leaves after mechanical wounding, insect infestation, and in response to Pseudomonas syringae pv. tomato infection (Chao et al. 1999; Gu et al. 1996b; Pautot et al. 1993). The potato Lap gene described by 
Hildmann et al. (1992) appears to be an analog of the tomato LapA genes.

Tomato genome has two highly conserved genes, LapAl and LapA2, encoding LAP-A proteins that are primarily controlled at the transcriptional level (Chao et al. 1999; Chao et al. 2000; Gu et al. 1996a). LapA genes are activated by the wound octadecanoid pathway because systemin and methyl jasmonate (MeJA) cause increases in the levels of LapA RNA, protein, and activity. In addition, salicylic acid (SA) suppresses wound induction of LapA genes (Chao et al. 1999), consistent with SA's role in blocking JA biosynthesis and JA action (Doares et al. 1995; Peña-Cortés et al. 1993).

Tomato pathogenesis-related protein $(P R)$ genes and wound-response genes (LapA, proteinase inhibitor 1 [pin1] and pin2) are induced in response to $P$. syringae pv. tomato (Jia and Martin 1999; Pautot et al. 1991; Pautot et al. 1993). The gene-for-gene resistance mechanism that is active in $P$. syringae pv. tomato-tomato interactions is one of the best characterized mechanisms (Martin 1999). P. syringae pv. tomato strains expressing the avirulence gene avrPto cause a hypersensitive disease resistance response in tomato lines expressing the disease resistance gene Pto. Biochemical and genetic studies have shown that during incompatible interactions, AvrPto and Pto interact directly to induce a signaling cascade involving transcription-factor phosphorylation, which leads to the rapid activation of $P R$ genes. In compatible interactions, pathogen recognition is slow and $P R$ gene expression is significantly delayed.

Superimposed on the activation of $P R$ genes are plant responses to the $P$. syringae pv. tomato phytotoxin coronatine. This toxin is responsible for the chlorotic halo that surrounds necrotic lesions on tomato plants (Mitchell and Young 1978). Coronatine plays a role in pathogen virulence (Bender et al. 1987) and has been proposed to mimic the action of MeJA/JA. A. thaliana mutants that are resistant to coronatine also are insensitive to MeJA (Feys et al. 1994) and MeJA/JA. Coronatine induces similar effects on plants, including inhibition root growth, stimulation of anthocyanin accumulation, and promotion of proteinase inhibitor accumulation (Bender et al. 1999; Feys et al. 1994; Koda et al. 1996; Sembdner and Parthier 1993; Wasternack et al. 1998; Weiler et al. 1994). Coronatine does not strictly mimic MeJA action, however, because this toxin induces other effects, including chlorosis and shrinking of chloroplasts not associated with MeJA treatments (Palmer and Bender 1995).

The induction of LapA by wounding and P. syringae pv. tomato infections seems incongruous given the reciprocal regulation of the wound-induced octadecanoid and the defenseresponse pathways (Walling 2000). To investigate the role of LAP-A in the defense response and during floral development, transgenic tomato plants expressing an antisense LapAl gene construct (35S:asLapA1) were evaluated. Levels of LapA RNAs, LAP-A and LAP-related proteins, and LAP-A activity were monitored during floral development and in response to mechanical wounding and $P$. syringae pv. tomato infection. The growth and development of Manduca sexta (tobacco hornworm) larvae on detached leaves from 35S:asLapA1 plants were also determined. In addition, the growth of $P$. $s y$ ringae pv. tomato coronatine-producing $\left(\mathrm{Cor}^{+}\right)$and -deficient (Cor ${ }^{-}$) strains in leaves of 35S:asLapA1 lines expressing the bacterial speck resistance gene (Pto) or its susceptible allele (pto) were evaluated. The role of coronatine in LapA gene expression also was evaluated by measuring changes in $L a p A$ RNAs, protein levels, and LAP-A activity in response to $\mathrm{Cor}^{+}$ and Cor $^{-}$P. syringae pv. tomato. To complement these studies, the impact of exogenous coronatine on the levels of LapA, pin2, and $P R$ gene $(P R-1$ and $P R-4)$ RNAs were assessed. Collectively, these results suggest that LapA genes were induced by wild-type $P$. syringae pv. tomato via a woundresponse pathway triggered by coronatine. These studies emphasized differences in exogenous coronatine and JA treatments on defense gene expression.

\section{RESULTS}

\section{Construction of a 35S:asLapA1 gene} and introduction into tomato plants.

A chimeric LapA1-antisense gene (35S:asLapA1) was constructed by inserting a 1.4-kb fragment of a LapAl cDNA (Pautot et al. 1993) between the Cauliflower mosaic virus (CaMV) 35S promoter with a double enhancer and the CaMV $35 \mathrm{~S}$ terminator. This chimeric gene was cloned into the Agrobacterium tumefaciens binary transformation vector pBIN19 (Bevan 1984). The recombinant A. tumefaciens strain was used to transform UC82b, a P. syringae pv. tomato-susceptible tomato cultivar. Six independent transgenic plants were selected on the basis of kanamycin resistance. Genomic DNA blot analysis and segregation analyses showed that the transgenic lines contained one to five copies of the 35S:asLapA1 gene at a single locus (data not shown).

\section{Accumulation of LapA transcripts in transgenic and control tomato lines.}

To determine whether the 35S:asLapA1 altered the levels of LapA RNAs that accumulated after wounding, RNAs were isolated from healthy and mechanically wounded leaves from the six $\mathrm{T}_{\mathrm{o}}$ 35S:asLapA1 transgenic lines and UC82b control plants. RNA blot analysis showed that LapA RNAs were not detected in healthy plants (Fig. 1A). After mechanical wounding, LapA RNAs accumulated to high levels in the

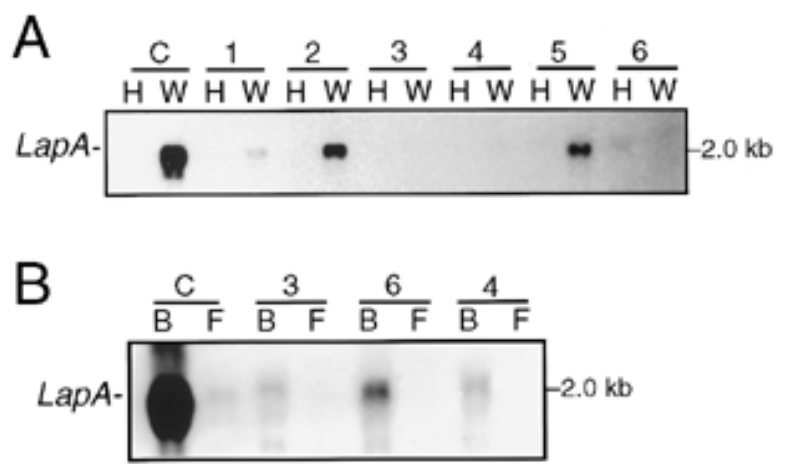

Fig. 1. Levels of LapA RNAs in control and transgenic tomatoes expressing the 35S:asLapA1 gene. A, Total RNAs were extracted from healthy $(\mathrm{H})$ and 20-h wounded (W) leaves of control (C) and six independent $\mathrm{T}_{\mathrm{o}}$ 35S:asLapA1 lines (1-6). B, Total RNAs were isolated from 8-mm green buds (B) and open flowers (F) from control (C) and 35S:asLapA1 plants 3, 4, and 6. Total RNAs (15 $\mu \mathrm{g} / \mathrm{lane})$ were fractionated, blotted, and hybridized to a ${ }^{32} \mathrm{P}$-labeled antisense LapAl RNA probe. The autoradiographs in $\mathbf{A}$ and $\mathbf{B}$ represent $15 \mathrm{~h}$ and $1 \mathrm{~h} 15 \mathrm{~min}$ exposures, respectively. The size of the LapA RNA is indicated in $\mathrm{kb}$. 
leaves of control plants. Three of the 35S:asLapAl lines (1, 2, and 5) accumulated LapA RNAs after wounding. The levels of the LapA RNAs, however. were reduced relative to the control plants. A stronger antisense inhibition of $L a p A$ gene expression was noted in the transgenic 35S:asLapA1 lines 3, 4, and 6. In these plants, the LapA RNAs were undetectable or barely detected in healthy and wounded leaves. These data indicated that the 35S:asLapA1 construct effectively down regulated the LapA1 and LapA2 genes.

Because LapA RNAs and proteins accumulate during floral development in tomato (Chao et al. 2000; Milligan and Gasser 1995), the levels of LapA RNAs in developing floral buds and open flowers were examined in the three transgenic 35S:asLapA1 lines $(3,4$, and 6) that showed the greatest reduction in leaf LapA RNA levels after wounding. In control plants, LapA RNAs were abundant in 8-mm green buds and were at low levels in open flowers (Fig. 1B). In contrast, LapA RNAs were detected at low-to-undetectable levels in the floral buds from 35S:asLapA1 plants. Transgenic lines 3 and 4 and their homozygous $\mathrm{T}_{2}$ progeny, which exhibited the most dramatic reduction in LapA RNA accumulation after wounding and during floral development, were further characterized.

\section{Accumulation of LAP proteins in the 35S:asLapA1 plants.}

Previous studies have revealed that tomato plants have four classes of proteins that are antigenically related to LAP (Gu et al. 1996b). There are two LAP-like proteins with molecular masses of 66 and $77 \mathrm{kDa}$ that are expressed constitutively and two classes of $55-\mathrm{kDa}$ LAPs with distinct pIs (Gu et al. 1996b). A single gene ( $L a p N)$ encodes the 55-kDa LAP-N polypeptides that have neutral pIs (6.6 to 7.0) and are expressed constitutively (Chao et al. 2000). Two genes (LapAl and LapA2) encode the 55-kDa LAP-A polypeptides that are wound induced and have acidic pIs (5.6 to 5.9) (Gu et al. 1996a). In addition to the epitopes shared between the LAP-A and LAP-N polypeptides (Gu et al. 1996b), the LapA genes and the single $\operatorname{LapN}$ gene-coding regions are $84 \%$ identical at the nucleotide level (C.-J. Tu and L. Walling, unpublished results). Given this sequence identity, it was important to assess the impact of 35S:asLapAl gene expression on the accumulation of LAP-A and LAP-N proteins.

To determine the levels of LAP and LAP-like proteins in the control UC82b plants and 35S:asLapAl lines, proteins were extracted from healthy and wounded leaves and fractionated by sodium dodecyl sulfate-polyacrylamide gel electrophoresis (SDS-PAGE). Gels were subjected to immunoblot analysis with a LAP polyclonal antiserum (Fig. 2A). Similar to the tomato cultivar Peto 238R (Gu et al. 1996b), the UC82b control plants had four immunoreactive proteins (Fig. 2A). The $90-\mathrm{kDa}$ protein was nonspecific and detected by the preimmune serum ( $\mathrm{Gu}$ et al. 1996b). The LAP antiserum also detected the 66- and 77-kDa LAP-like proteins and low levels of a 55-kDa LAP protein in healthy UC82b leaves. After wounding, the levels of the 66- and 77$\mathrm{kDa}$ polypeptides remained constant and the levels of the $55-\mathrm{kDa}$ LAP proteins rose markedly. When the $\mathrm{T}_{2}$ progeny from the transgenic 35S:asLapAl lines 3 and 4 were examined, only the nonspecific $90-\mathrm{kDa}$ and the 66- and $77-\mathrm{kDa}$ LAP-like proteins were detected (Fig. 2A). The 55-kDa LAP proteins were not detected in either healthy or wounded transgenic leaves.
Total proteins from healthy and wounded leaves from control and 35S:asLapA1 plants were fractionated by two dimensional (2D)-PAGE. Immunoblots were incubated with the LAP polyclonal antiserum (Fig. 2B and C). Wounded leaves from the control plants accumulated the 66- and 77-kDa LAPlike proteins as well as the LAP-N and LAP-A polypeptides (Fig. 2B). In contrast, only the 66- and 77-kDs LAP-like proteins were detected in the wounded leaves from the 35S:asLapA1 lines. Neither the constitutive LAP-N nor the wound-induced LAP-A proteins were detected in wounded leaves from the 35S:asLapA1 lines. Some variation in the degree of down regulation of LAP-A and LAP-N imposed by the 35S:asLapA1 gene was noted in repeated experiments. In all cases, however, LAP-A and LAP-N proteins were either undetectable (Fig. 2B) or at low levels (data not shown) in wounded leaves.

Given the role of aminopeptidases in protein turnover (Taylor 1996) and the reduction of the LAP-A and LAP-N polypeptides, it was interesting to determine whether the 35S:asLapA1 plants exhibited differences in their total protein profiles. Total proteins were extracted from healthy and
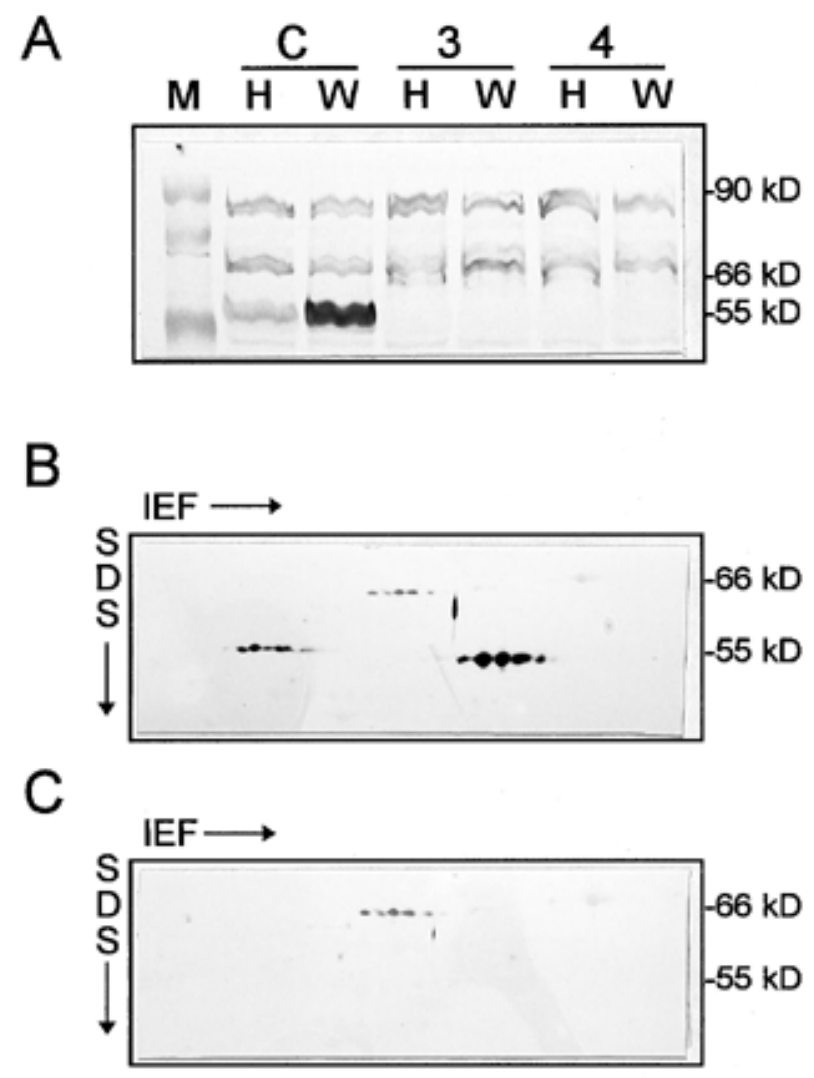

Fig. 2. Accumulation of LAP and LAP-like proteins in leaves of control and 35S:asLapA1 transgenic plants. Total proteins were isolated from healthy $(\mathrm{H})$ and wounded $(\mathrm{W})$ leaves from control and homozygous $\mathrm{T}_{2}$ 35S:asLapA1 plants (lines 3 and 4). A, Total proteins $(25 \mu \mathrm{g})$ were fractionated by sodium dodecyl sulfate-polyacrylamide gel electrophoresis (SDS-PAGE). B and C, Total proteins $(80 \mu \mathrm{g})$ from wounded leaves from control (B) and 35S:asLapA1 plants (C) were fractionated by twodimensional (2D)-PAGE. SDS- and 2D-PAGE immunoblots were incubated with a 1:500 dilution of the LAP polyclonal antiserum. Molecular weight markers $(\mathrm{M})$ were run in parallel lanes and are indicated in $\mathrm{kD}$. Data for line 4 plants are shown; similar results were found with line 3 plants. 
wounded leaves from control and 35S:asLapA1 plants, fractionated by 2D-PAGE, and stained with silver. Comparison of protein profiles from the control and 35S:asLapA1 plants showed no reproducible differences in healthy and wounded leaves, except for the presence of LAP-A in wounded leaves from the control (Fig. 3).

\section{Impact of LAP down regulation} on floral and fruit development.

Given the high levels of LAP proteins and activity in developing flowers from control plants (Fig. 1B) (C.-J. Tu and L. Walling, unpublished results), the impact of LAP-A and LAP$\mathrm{N}$ down regulation on floral development and function was investigated. Floral structure was not altered in 35S:asLapA1 plants, and no significant differences in the number and weight of fruit or the number of seeds per fruit were observed in the control and 35S:asLapA1 lines (data not shown).

\section{Influence of LAP down regulation on $P$. syringae $\mathrm{pv}$.} tomato-compatible and -incompatible interactions.

Leaves from the control and 35S:asLapA1 transgenic plants were inoculated with $2 \times 10^{6} \mathrm{CFU} / \mathrm{ml}$ of $P$. syringae pv. to- mato PT11 (compatible interaction). The levels of LAP-A enzymatic activity and LapA RNA were monitored 0, 2, 3, and 4 days after $P$. syringae pv. tomato inoculation (Fig. 4). $P$. syringae pv. tomato infection caused LapA RNA and LAP-A activity levels to rise in the leaves of control UC82b plants after 2 days. While LAP-A activity persisted in leaves 4 days after $P$. syringae pv. tomato inoculation, LapA RNA levels declined by day 4 . In contrast to the control-line response, no LAP-A activity was detected (Fig. 4A) and the accumulation of LapA RNA (Fig. 4B) was greatly reduced in infected leaves from the 35S:asLapA1 plants. Similar results were obtained in transgenic line 3 (data not shown).

Despite the down regulation of LapA RNAs, the LAP-A and LAP-N proteins, and LAP activities, the 35S:asLapA1 lines and the control plants did not differ in the number and size of the $P$. syringae pv. tomato-induced lesions (data not shown). To determine if $P$. syringae pv. tomato growth rates were influenced, the impact of the 35S:asLapAl gene on intercellular bacterial titers during compatible and incompatible interactions was determined. During the compatible interaction with the disease-susceptible UC82b (pto) and 35S:asLapAl line 4
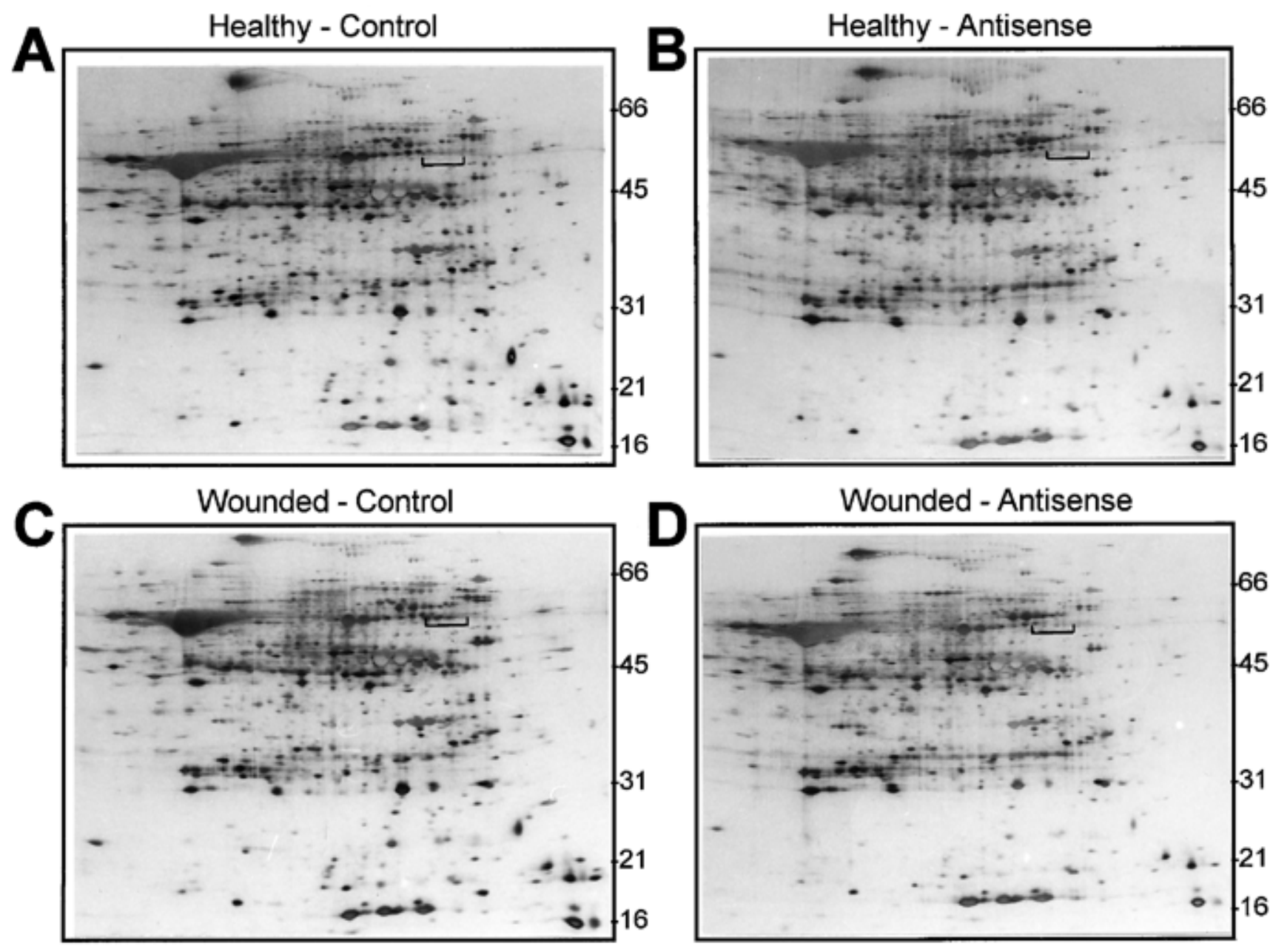

Fig. 3. Total protein profiles in control and 35S:asLapA1 plants. Total proteins $(80 \mu \mathrm{g})$ were isolated from healthy (A and B) and wounded (C and D) leaves from control and homozygous $\mathrm{T}_{2}$ 35S:asLapA1 plants (line 4). Proteins were fractionated by two-dimensional polyacrylamide gel electrophoresis (2D-PAGE), and gels were silver stained. Molecular weight markers were run in parallel lanes and are indicated in $\mathrm{kD}$. The positions of wound-induced LAP-A proteins are indicated by a bracket in $\mathbf{C}$. Brackets also were added in $\mathbf{A}, \mathbf{B}$, and $\mathbf{D}$ to emphasize the absence of LAP-A proteins in the samples. The left and right ends of the $2 \mathrm{D}$ gel represent $\mathrm{pH} 8$ to 4 , respectively. 
(pto), P. syringae pv. tomato PT11 levels increased during the 4-day period and reached peak levels on day 4 (Fig. 5A). $P$. syringae pv. tomato grew at similar rates in the control and 35S:asLapA1 lines. To examine the impact of LapA and LapN down regulation on $P$. syringae pv. tomato PT11 growth during an incompatible interaction, two disease-resistant lines (Pto and 35S:asLapA1 Pto) were infected with P. syringae pv. tomato (Fig. 5B). The intercellular titers of $P$. syringae pv. tomato PT11 during the compatible interactions were over 550 -fold higher than with incompatible interactions. Decreased levels of LAP-A and LAP-N did not significantly influence bacterial growth in disease-resistant plants.

\section{Effect of 35S:asLapA1 on insect growth.}

Larvae of $M$. sexta were fed a diet consisting of leaves from either control or 35S:asLapA1 transgenic plants to assess the effect of LAP-A and LAP-N on insect growth. Over 21 larvae were monitored on each class of plant. The mass and the developmental stage of the larvae were noted after 9 and 12 days of feeding. No significant difference in larvae growth was observed between the control and 35S:asLapAl transgenic lines 3 and 4 (data not shown).

\section{Aminopeptidase activity and levels of $\operatorname{LapA}$ and pin2 RNAs in response to coronatine-producing and -deficient $P$. syringae pv. tomato strains.}

Previous studies have shown that LapA, pin1, and pin2 RNAs accumulate in tomato leaves during compatible and incompatible interactions with the coronatine-producing $P$. syringae pv. tomato strain PT11 (Pautot et al. 1991; Pautot et
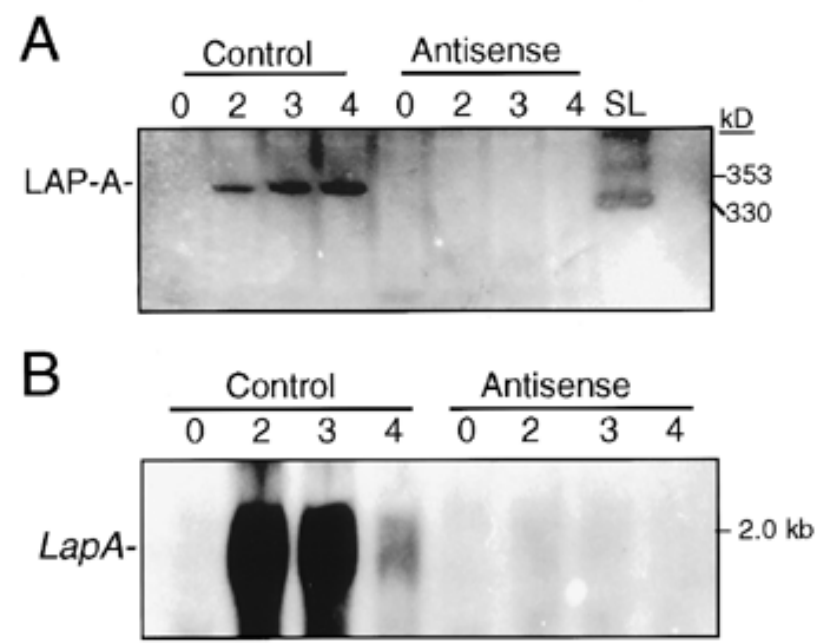

Fig. 4. Accumulation of LAP-A activity and LapA1 RNAs in leaves of control and 35S:asLapA1 plants during compatible Pseudomonas syringae pv. tomato interactions. Native proteins or RNAs were extracted from control and 35S:asLapA1 plant leaves 0, 2, 3, and 4 days after $P$. syringae pv. tomato inoculation. A, Soluble tomato proteins $(40 \mu \mathrm{g} / \mathrm{lane})$ and swine LAP (SL; $0.5 \mu \mathrm{g} / \mathrm{lane}$ ) were fractionated by native polyacrylamide gel electrophoresis, and gels were stained for aminopeptidase activity with leucine- $\beta$-naphthylamide and Fast Garnet GBC (Gu et al. 1996b). The masses of the tomato LAP-A hexamer (353 kDa) and the swine LAP hexamer $(330 \mathrm{kDa})$ were determined previously $(\mathrm{Gu}$ et al. 1996b). B, Total RNA blots $(15 \mu \mathrm{g})$ were hybridized to a ${ }^{32} \mathrm{P}$-labeled antisense LapA1 RNA probe. The autoradiograph represents a 1-h exposure. The size of the LapA RNA is indicated in $\mathrm{kb}$ al. 1993). To evaluate the induction of LapA and pin2 RNAs by coronatine during compatible interactions, leaves from control (UC82b) plants were inoculated with the $\mathrm{Cor}^{+}$or $\mathrm{Cor}^{-}$ strains of $P$. syringae pv. tomato. LAP activity was detected on day 3 after inoculation with the $\mathrm{Cor}^{+}$strain PT11, whereas LAP activity was not detected over the 4-day infection period with the $\mathrm{Cor}^{-}$mutant PT23.2 (Fig. 6A). Similar findings were found at the RNA level (Fig. 6B). LapA and pin2 RNAs were present at high levels in leaves after infection with the $\mathrm{Cor}^{+}$ strain of $P$. syringae pv. tomato and were not detected after infection by the $\mathrm{Cor}^{-}$strain.

To confirm these data, excised seedling shoots were treated with $1 \mu \mathrm{mol}$ of coronatine. The levels of transcripts for several tomato wound- and defense-response genes were determined at $0,2,4,8$, and $16 \mathrm{~h}$ after coronatine treatment (Fig. 7). LapA and pin2 RNAs accumulated in response to exogenous coronatine. For comparison, JA-responsive $(P R-1)$ and nonresponsive $(P R-4) P R$ gene RNA levels were assessed (Chao et al. 1999). $P R-4$ and $P R-1$ RNAs were not detected or were at very low levels after $16 \mathrm{~h}$ of coronatine treatment.

\section{Growth of Cor $^{-} P$. syringae pv. tomato in control and 35S:asLapA1 plants.}

Coronatine is a strong inducer of LapA genes of tomato (Fig. 7). To test whether differences in P. syringae pv. tomato growth rates could be observed with coronatine-deficient $P$. syringae pv. tomato, the rate of growth of $P$. syringae pv. tomato strain PT23.2 during compatible and incompatible infections of control and 35S:asLapA1 plants was determined. Like the coronatine-producing strain PT11, PT23.2 intercellular titers rose to similar levels in compatible infections of control (UC82b) and 35S:asLapA1 plants (Fig. 5C). The $P$. syringae pv. tomato titer on day 4 was substantially lower (40to 150 -fold) than observed for the $\mathrm{Cor}^{+}$strain (Fig. 5A and C). This could be attributed partially to the 1.5 -fold-lower inoculation concentration of the $\operatorname{Cor}^{-} P$. syringae pv. tomato used in these infections. This difference, however, is more likely attributed to the reduced fitness of coronatine-deficient $P$. syringae pv. tomato (Bender et al. 1987). The ability of PT23.2 to grow in control and 35S:asLapAl plants harboring the Pto resistance gene also was assessed (Fig. 5D). Similar to the Cor $^{+}$PT11 during incompatible interactions, intercellular $P$. syringae pv. tomato PT23.2 titers were substantially less (6- to 20-fold, reduced) relative to compatible interactions (Fig. 5C and D). No differences in PT23.2 growth rates were observed in the Pto or 35S:asLapA1 Pto lines (Fig. 5D). The reduced fitness that was clearly observed during PT23.2 Cor-compatible interactions was not observed as clearly in the incompatible interactions.

\section{DISCUSSION}

These studies have investigated the role of LAP-A in the tomato defense response and during floral development. Previous studies showed that LapAl and LapA2 mRNAs encoding LAP-A proteins were induced in response to mechanical wounding, insect infestation, and P. syringae pv. tomato infection (Chao et al. 1999; Gu et al. 1996b; Pautot et al. 1993). In healthy plants, LapA mRNAs accumulated at high levels in closed floral buds. Here an antisense RNA strategy was exploited to gain information about the role of 
LAP-A in tomato development and defense responses. Two tomato 35S:asLapA1 lines that displayed greatly reduced levels of LapA RNAs and no detectable LAP-A activity in floral buds and after wounding were chosen for further analysis.

Immunoblot analysis with LAP-A polyclonal antibodies indicated that the 35S:asLapAl plants with genes encoding the acidic (LapAl and LapA2) and neutral (LapN) LAPs were down regulated. These data indicated that the chimeric 35S:asLapA1 gene down regulated the wound-induced LapA genes (LapA1 and LapA2) and the constitutively expressed LapN gene. Comparisons of the LapAl region in the 35S:asLapA1 construct with the corresponding $L a p N$ region indicated that the LapAI and LapN genes were $84 \%$ identical at the nucleotide level. A few short blocks of $100 \%$ nucleotide sequence identity (40 bp) also were located in this region (C.-J. Tu and L. Walling, unpublished results). The coordinate down regulation of the LapA and LapN genes suggest that antisense RNAs can tolerate a moderate percentage of sequence mismatch and still be effective. Similar observations were made with the Petunia hybrida chalcone synthase gene family (Van der Krol et al. 1990). In fact, the regions with absolute identity may be critical in determining the success of an antisense strategy because Cannon et al. (1990) showed that a 41-bp region with $100 \%$ identity was sufficient to impart an antisense effect.

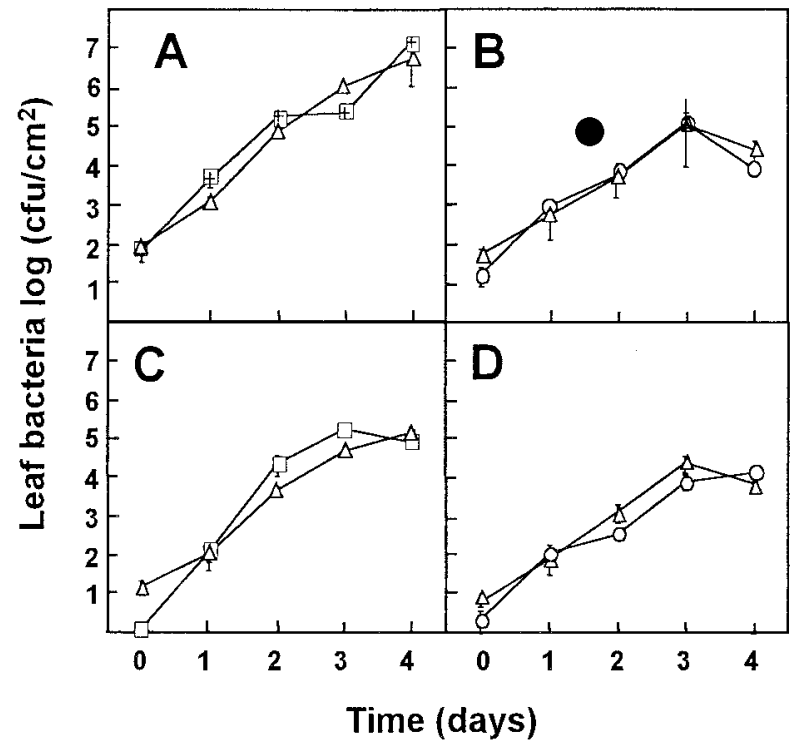

Fig. 5. Growth of Pseudomonas syringae pv. tomato in control and 35S:asLapA1 transgenic plants. Leaves from 3.5-week-old control and 35S:asLapA1 lines were inoculated with $3.8 \times 10^{6} \mathrm{CFU} / \mathrm{ml}$ of $P$. syringae pv. tomato PT11 $\left(\mathrm{Rif}^{\mathrm{R}} \mathrm{Cor}^{+}\right)\left(\mathrm{A}\right.$ and B) or $2.5 \times 10^{6} \mathrm{CFU} / \mathrm{ml} P$. syringae pv. tomato PT23.2 ( iff $\left.^{\mathrm{R}} \mathrm{Cor}^{-}\right)(\mathbf{C}$ and $\mathbf{D})$. The number of $P$. syringae pv. tomato colony-forming units per $\mathrm{cm}^{2}$ was monitored over a period of 4 days after inoculation. Each value is the mean titer from four leaf discs from three different plants of each line. Standard error bars are indicated. A, Growth of PT11 during compatible interactions in the UC82b control (open triangles) and 35S:asLapAl lines (closed squares). B, Growth of PT11 during incompatible interactions in the Pto238R (open circles) and 35S:asLapA1 Pto lines (closed triangles). C, Growth of PT23.2 during compatible interactions in the UC82b control (open triangles) and 35S:asLapA1 lines (closed squares). D, Growth of PT23.2 during incompatible interactions in the Pto238R (open circles) and 35S:asLapA1 Pto lines (closed triangles).
Because LapA mRNAs increase in incompatible and compatible $P$. syringae pv. tomato interactions (Pautot et al. 1993), the impact of LapA down regulation on compatible and incompatible interactions was examined. These experiments indicated that the greatly reduced levels of LAP-A and LAP-N did not significantly impact P. syringae pv. tomato intercellular growth rates during compatible and incompatible interactions. The severity of the symptoms, number and size of lesions, and growth rate of $P$. syringae pv. tomato in leaves of 35S:asLapAl pto and 35S:asLapA1 Pto relative to their control plants were similar. Additionally, the LAP-A and LAP-N deficiency did not alter the growth of M. sexta larvae. Studies in potato showed that down regulation of the potato Lap gene did not interfere with Phytophthora infestans growth (Herbers et al. 1994).

Like pathogen invasion, the role of LAP-A during floral development remains unclear. The levels of LapA RNAs and LAP-A proteins in closed tomato buds and in the ovary integument, in particular, are high (Chao et al. 1999; C.-J. Tu and L. Walling, unpublished results). The transgenic 35S:asLapA1 tomato lines that had greatly reduced levels of LAP-A and LAP-N exhibited no obvious phenotypic alterations in floral structure and were fully fertile. Similar results were observed when the potato Lap antisense RNA was expressed in transgenic potato plants (Herbers et al. 1994). These data suggested that the tomato LAPs might not play a critical role in floral structure and reproductive development.
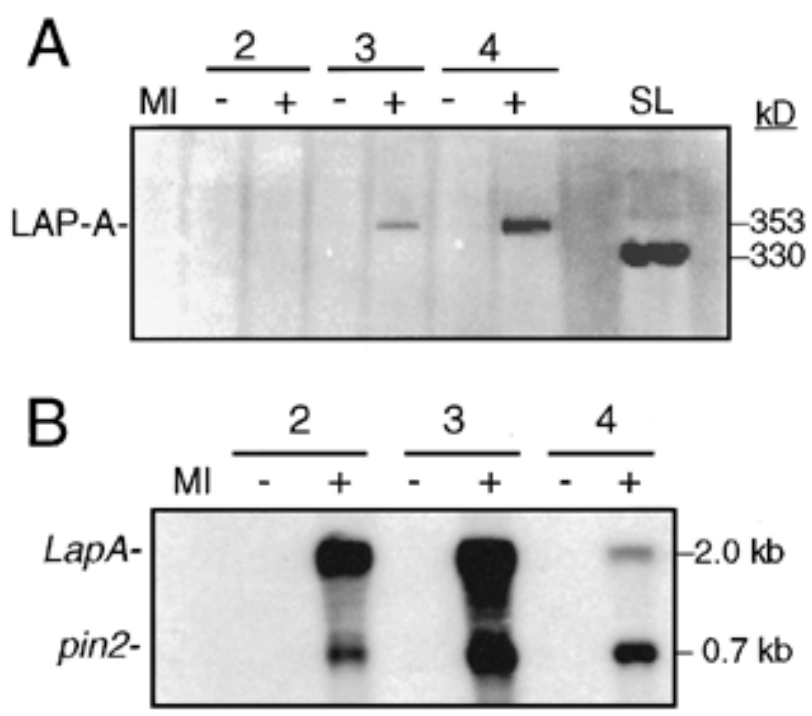

Fig. 6. Accumulation of LAP-A activity and LapA RNAs in response to wild-type $\left(\mathrm{Cor}^{+}\right)$and coronatine mutant $\left(\mathrm{Cor}^{-}\right)$strains of Pseudomonas syringae pv. tomato . Leaves of 3-week-old UC82b plants were mockinfected (MI), or inoculated with $2 \times 10^{7} \mathrm{CFU} / \mathrm{ml}$ of $P$. syringae pv. tomato PT11 ( $\mathrm{Cor}^{-}$, minus symbol) or PT23.2 ( $\mathrm{Cor}^{+}$, plus symbol). Leaves from mock-inoculated and $\mathrm{Cor}^{+}$- or $\mathrm{Cor}^{-}$-compatible interactions were harvested $0,2,3$, and 4 days later. A, Native proteins were extracted from leaves, and $40 \mu \mathrm{g}$ of soluble protein was fractionated by native polyacrylamide gel electrophoresis. The swine LAP (SL; $0.5 \mu \mathrm{g} / \mathrm{lane}$ ) was used as control. LAP-A activity was measured by the in situ gel assay, as described in Gu et al. (1996b). Masses of the tomato LAP-A and swine LAP were determined previously (Gu et al. 1996b) and are shown in $\mathrm{kD}$. B, Total RNAs were extracted from leaves. Total RNA blots (15 $\mu \mathrm{g}$ per lane) were hybridized to ${ }^{32} \mathrm{P}$-labeled LapA1 and pin 2 probes. The sizes of LapA and pin2 RNAs are indicated in $\mathrm{kb}$. Autoradiograms were exposed for $2 \mathrm{~h}$. 
It is possible that minute quantities of active LAP-A and LAP-N accumulated in the 35S:asLapAl lines and were sufficient to catalyze their role in development and in curtailment of pathogen invasion or deterring caterpillar growth or feeding. Alternatively, the impact of LapA and LapN down regulation on plant defense and floral development also could be masked by other exopeptidases that have substrate specificities similar to LAP-A and/or LAP-N. For example, the constitutively expressed tomato-leaf aminopeptidase (AMP1) has a substrate specificity that overlaps with LAP-A. LAP-A and AMP1 hydrolyze substrates with N-terminal leucine (Leu), methionine (Met), and alanine (Ala) residues (Gu and Walling 2000; Gu et al. 1999; N. Ly and L. Walling, unpublished results). It is clear that a more comprehensive understanding of the complement of aminopeptidases in healthy and stressed tomato leaves will be necessary to thoroughly evaluate the role of LAP-A and LAP-N in development and in response to stress. Our relative lack of knowledge in this area is indicated by the fact that there have been few comprehensive surveys for developmentally regulated or defense-response aminopeptidases (Walling and $\mathrm{Gu}$ 1996). The observation that the aminopeptidase inhibitor, bestatin, activates the tomatowound response suggests a negative regulatory role for a bestatin-sensitive peptidase in the tomato-defense response (Schaller and Ryan 1996) and emphasizes the need for further studies of peptidases in plant-stress responses. Analysis of transgenic tomato plants that are cosuppressed for LapA expression and express a LapA:asLapA construct and overexpress $L a p A$ are being examined for their impact on pathogen and herbivore interactions as well as their roles in protein and peptide turnover.

While LapA RNAs accumulated in leaves in response to $P$. syringae pv. tomato (Pautot et al. 1993) and the fungal root pathogen Phytophthora parasitica (N.-S. Jwa and L. Walling,

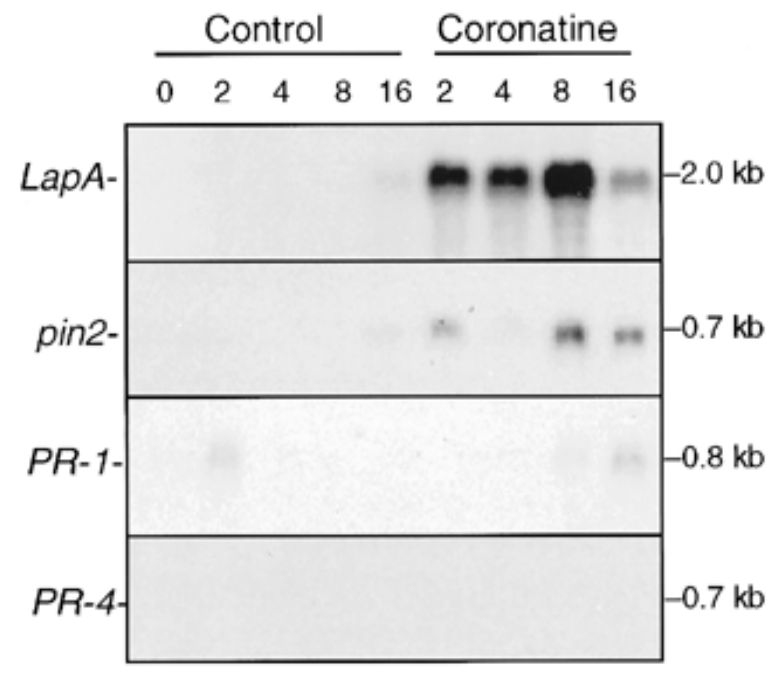

Fig. 7. Coronatine RNA blots. Shoots were excised from 3-week-old seedlings and $1 \mu \mathrm{mol}$ of coronatine in $0.002 \%$ ethanol was applied to excised seedlings. Control plants were treated with $0.002 \%$ ethanol in a similar manner. Leaves were harvested $0,2,4,8$, and $16 \mathrm{~h}$ after treatment. Total RNAs were extracted from leaves. Total RNA blots (15 $\mu \mathrm{g}$ per lane) were hybridized to ${ }^{32} \mathrm{P}$-labeled LapA1, pin2, PR-1, and $P R-4$ probes. The sizes of RNAs are indicated in $\mathrm{kb}$. The LapA1, pin2, $P R-1$, and $P R-4$ RNA blots were exposed to film for $72 \mathrm{~h}$. unpublished results), the foliar pathogen Xanthomonas campestris pv. vesicatoria did not induce LAP-A activity (data not shown). In addition, potato LapA RNAs did not accumulate in response to $X$. campestris pv. vesicatoria or $P$. infestans (Herbers et al. 1994). The fact that i) there is nonuniform induction of LapA by pathogens, ii) LapA RNAs accumulate with a similar timing in compatible and incompatible interactions with $P$. syringae pv. tomato (Pautot et al. 1993), and iii) LapA genes are induced by the wound signals MeJA and systemin (Chao et al. 1999) prompted further investigations of the mechanisms underlying the induction of LapA by $P$. syringae pv. tomato. One feature that distinguishes $P$. syringae pv. tomato from the other pathogens mentioned is its ability to produce the phytotoxin coronatine (Bender et al. 1999). Coronatine mimics some of the responses induced by MeJA (Bender et al. 1999; Koda et al. 1996; Wasternack et al. 1998; Weiler et al. 1994), and appears to use similar mechanisms for perception because $A$. thaliana mutants that are resistant to coronatine are also insensitive to MeJA (Feys et al. 1994).

The expression of the tomato LapA and pin2 genes in response to $\mathrm{Cor}^{+}$and $\mathrm{Cor}^{-}$strains of $P$. syringae pv. tomato was evaluated. LAP-A activity, LapA RNAs, and pin2 RNAs accumulated to high levels with the $\operatorname{Cor}^{+}$strain but not with the Cor $^{-}$strain. These data were supported by studies treating excised tomato seedlings with coronatine, indicating that the changes in LapA and pin2 gene expression in response to $P$. syringae pv. tomato were mediated primarily by the coronatine. Similar results for pin2 RNA accumulation in response to coronatine treatments were consistent with coronatine acting as a JA mimic, as reported by Wasternack et al. (1998). Previous studies showed that systemin, a peptide activator of the wound octadecanoid pathway, and MeJA cause LapA and pin2 RNAs to accumulate (Chao et al. 1999; Farmer and Ryan 1990; Pearce et al. 1991; Wasternack et al. 1998).

The responses of two $P R$ genes to coronatine also were examined. The tomato $P R-4$ transcripts did not accumulate in response to MeJA (Chao et al. 1999) or coronatine. Surprisingly, $P R-1$ RNAs accumulated in response to exogenous MeJA (Chao et al. 1999), yet coronatine treatments were insufficient to cause a large increase in $P R-1$ transcript levels. These data indicated that, despite similarities in many coronatine and MeJA-induced responses (Koda et al. 1996; Wasternack et al. 1998; Weiler et al. 1994), coronatine and MeJA act in a distinct manner in the excised seedling assay. The in vivo expression of wound- and defense-response genes often is separated temporally as a result of reciprocal regulation of the wound-octadecanoid pathway and defenseresponse pathways by MAP kinases, small GTP-binding proteins, and SA (Doares et al. 1995; Doherty et al. 1988; PeñaCortés et al. 1993; Romeis et al. 1999; Sano et al. 1994; Seo et al. 1995). For example, Mittal et al. (1995) showed that Cor $^{+}$ $P$. syringae pv. tomato delayed expression of $A$. thaliana defense-response genes relative to $\operatorname{Cor}^{-} \mathrm{P}$. syringae $\mathrm{pv}$. tomato strains. Coronatine and systemin treatments retain the cross talk that coordinates the wound- and defense-response signaling pathways because both treatments induce woundresponse genes such as LapA and pin2 but not PR genes (Fig. 6 and Table 1) (Chao et al. 1999). In contrast, MeJA treatments do not retain this specificity because exogenous MeJA treatments induce both wound-response genes (LapA and pin2) as well as JA-regulated $P R$ genes $(G l u B, C h i 9$, and $P R$ - 
1) (Chao et al. 1999; D. Puthoff, C. LeVesque, W. Chao, T. Perring, and L. Walling, unpublished results).

Although the signal transduction pathways that are responsible for mediating coronatine and MeJA inhibition of root growth and wound response overlap (Feys et al. 1994; Rojo et al. 1999), the data presented here shows that there must be elements of the wound- and defense-signaling pathways that respond to exogenous MeJA and coronatine differently. One interpretation is that MeJA treatments did not produce a regulator that suppressed the defense pathway, thereby leading to up regulation of $P R-1$, and that coronatine and systemin treatments may have produced this regulator. Alternatively, coronatine and systemin may have induced an inhibitor of JAregulated $P R-1$ expression or prevented the accumulation of a defense-response factor critical for its expression. It also is possible that the JA that accumulated after systemin treatments and the coronatine that was taken up in the seedling assays may be compartmentalized and therefore not impact cells that can express $P R$ genes (Chao et al. 1999; Harms et al. 1995; Schaller and Ryan 1996). Perhaps MeJA and/or coronatine were transported or catabolized at different rates in different populations of cells, allowing coronatine to solely induce wound-response genes and MeJA to activate woundand defense-response genes. These surprisingly complex interactions stress that intricate networks are utilized to coordinate and prioritize the multiple signal transduction pathways active in response to pathogens and pests (Dempsey et al. 1999; Pieterse and van Loon 1999; Ryan 2000; Walling 2000).

\section{MATERIALS AND METHODS}

\section{Construction of transgenic tomatoes expressing 35S:asLapA1.}

The 1.4-kb EcoRI-SpeI fragment containing $81 \%$ of the LapAl coding region (nucleotides 324 to 1,736 in the LapAl cDNA sequence; GenBank accession no. U50151) was used to design a LapAl antisense construct. This fragment was shuttled into pBluescript-KS+ to facilitate further manipulations. The LapAl fragment was excised from pBluescript-KS+ with $X b a \mathrm{I}$ and $E c o \mathrm{RI}$ and subcloned into pUC18. The pUC18 clone was digested with EcoRI and SalI. The LapA fragment was cloned in the antisense orientation between the CaMV 35S promoter with a double enhancer and the transcriptional termination region from the CaMV 35S gene in the pLBR19 plasmid vector (T. Michael, Norwich Research Park, Colney, Norwich, U.K.). pLBR19 was derived pJIT60 (Guerineau et al. 1992) and the EcoRI site of pJIT60 was abolished by adding an $\mathrm{XbaI}$ linker. The chimeric 35S:asLapAl gene was excised with $X h o I$ and $K p n I$ and ligated into the binary transformation vector pBin19 hydrolyzed with KpnI and SalI (Bevan 1984). This construct was introduced into A. tumefaciens C58 (An et al. 1988).

Conditions for plant and A. tumefaciens growth and procedures for tomato transformation have been described (Hamza and Chupeau 1993). Lycopersicon esculentum Mill. cv. UC82b (Harris Moran Seed Co., Salinas, CA, U.S.A.) cotyledons were infected with A. tumefaciens (35S:asLapAl). Plants were regenerated in the presence of kanamycin. The presence of the 35S:asLapAl transgene in $\mathrm{T}_{\mathrm{o}}$ lines 1 to 6 was detected by genomic DNA blot analysis. Homozygous $\mathrm{T}_{2}$ progeny from lines 3 and 4 were identified. The 35S:asLapAl plants, similar to their parental line UC82b, were susceptible to $P$. syringae pv. tomato infection (pto allele).

Homozygous 35S:asLapAl pto (line 4) plants were used as the female parent in a cross with a homozygous Pto line (Peto238R). Peto238R is an inbred tomato line with the Pto resistance gene, which confers resistance to $P$. syringae pv. tomato race 0 strains. Peto238R was initially provided by $\mathrm{J}$. Williams (Petoseed Co., Saticoy, CA, U.S.A.). $F_{1}$ and $F_{2}$ progeny expressing the Pto gene were identified by screening for resistance $72 \mathrm{~h}$ after inoculation of leaves with $5 \times 10^{6}$ $\mathrm{CFU} / \mathrm{ml}$ P. syringae pv. tomato, as described previously (Stockinger and Walling 1994). The presence of the Pto allele was confirmed with allele-specific primers (Pto2 primer: $5^{\prime}$-T GCTTTAaGCTCGAGTTATC-3'; Pto5 primer: 5'-TCCCTTG GAAGAGATTGAACT). The presence of the 35S:asLapAl transgene was followed with primers to the linked kanamycin resistance gene (Npt-F primer: 5'-CCGCGTACCTGCCCATT C-3'; Npt-R primer: 5'-GCGATAGAA-GGCGATGCG-3'). Annealing temperatures for the Pto and nptII primers were 52 and $63^{\circ} \mathrm{C}$, respectively. Polymerase chain reaction conditions have been described previously (Van de Ven et al. 2000). A homozygous 35S:asLapAl Pto plant was identified and used in $P$. syringae pv. tomato infection studies.

\section{P. syringae pv. tomato infections, wounding, and coronatine treatments.}

Control and 35S:asLapAl transgenic tomato plants were grown in a greenhouse under day-night temperatures of $30^{\circ} \mathrm{C}$ (day), $15^{\circ} \mathrm{C}$ (night), or in growth chambers with a 16-h day cycle at 27 to $28^{\circ} \mathrm{C}$ and an 8 -h night cycle at 18 to $20^{\circ} \mathrm{C}$. Completely open flowers and 8 -mm green buds were harvested from greenhouse-grown plants. Tissues used for LAP activity assays were used immediately; tissues used for RNA extractions were frozen in liquid nitrogen and stored at $-80^{\circ} \mathrm{C}$ until use. Methods for $P$. syringae pv. tomato inoculation, wounding, tissue harvest, and tissue storage have been described previously (Pautot et al. 1991).

A rifampicin-resistant $P$. syringae pv. tomato PT11 was selected by their ability to grow on King's B media plates containing $50 \mu \mathrm{g}$ of rifampicin per $\mathrm{ml}$ and $0.2 \%$ ( $\mathrm{vol} / \mathrm{vol}$ ) dimethyl sulfoxide (DMSO). PT11 $\left(\mathrm{Rif}^{\mathrm{R}}\right)$ produces the phytotoxin coronatine $\left(\mathrm{Cor}^{+}\right)$, whereas PT23.2 $\left(\mathrm{Rif}^{\mathrm{R}}\right)$ is defective in coronatine production $\left(\mathrm{Cor}^{-}\right)$. For compatible interactions, $P$. syringae pv. tomato was inoculated on control (UC82b) and 35S:asLapA1 leaves. For incompatible interactions, P. syringae pv. tomato was inoculated on control (Peto238R Pto) and 35S:asLapA1 Pto leaves. Leaves from 3.5-week-old control and 35S:asLapA1 plants were inoculated with $3.8 \times 10^{6}$ $\mathrm{CFU} / \mathrm{ml}$ of the $P$. syringae pv. tomato $\mathrm{Cor}^{+}$and $2.5 \times 10^{6}$ $\mathrm{CFU} / \mathrm{ml}$ of the $\mathrm{Cor}^{-}$strains. At various times after inoculation, two leaves were harvested from each inoculated plant and four disks $\left(0.78 \mathrm{~cm}^{2}\right)$ were excised. Disks from a single plant were

Table 1. Expression of wound- and defense-response genes.

\begin{tabular}{lccc}
\hline & \multicolumn{3}{c}{ Treatments } \\
\cline { 2 - 4 } Gene & Systemin & Coronatine & MeJA \\
\hline LapA & + & + & + \\
pin2 & + & + & + \\
$P R-1$ & - & - & + \\
$P R-4$ & - & - & - \\
\hline
\end{tabular}


pooled and three independent plants were assessed per time point. Four disks were rinsed three times in $50 \mathrm{ml}$ of $1 \times$ phosphate-buffered saline (PBS) $(170 \mathrm{mM} \mathrm{NaCl} ; 6.2 \mathrm{mM} \mathrm{KCl}$; $12.6 \mathrm{mM}$ Na2HPO4; $2.2 \mathrm{mM}$ KH2PO4, $\mathrm{pH}$ 7.4). Leaf disks were homogenized in $1 \times$ PBS and the homogenate was serially diluted in $1 \times$ PBS to determine the number of colony forming units per milliliter. Titers of rifampicin-resistant $P$. syringae pv. tomato were determined on King's B medium with $50 \mu \mathrm{g}$ of rifampicin per $\mathrm{ml}$ and $0.2 \%$ DMSO.

For coronatine treatments, 3-week-old tomato plants were excised at the base of the shoot. A $90-\mu$ l drop of $1 \mu \mathrm{mol}$ of coronatine in $0.002 \%$ ethanol was applied to the shoot. After 3 to $4 \mathrm{~min}$, the drop was taken up by the transpiration stream and shoots were placed in water for 2 to $16 \mathrm{~h}$. Control seedlings were fed $90 \mu \mathrm{l}$ of $0.002 \%$ ethanol.

\section{Insect feeding experiments.}

M. sexta eggs were obtained from Plant Genetic Systems (Gent, Belgium). The greatest survival rate for larvae occurred when new emerging larvae were fed on leaves. Raising larvae on an artificial diet prior to transfer to control or 35S:asLapA1 tomato plants decreased survival significantly. For this reason eggs were placed on detached leaves from 35S:asLapAl plants that did not accumulate or had low levels of LAPs and were incubated at $25^{\circ} \mathrm{C}$. Seven 2- to 3-day-old first-instar larvae (6 mg each) were selected and placed on each 5-weekold control and 35S:asLapA1 plant in insect cages in the greenhouse. Leaves from six independent plants of each transgenic line were used in feeding trials. After 9 and 12 days, surviving larvae were removed, their developmental stages determined, and their masses recorded. Larvae survival rate on control and antisense plants was $50 \%$.

\section{RNA isolation and RNA blot hybridizations.}

Procedures for the isolation of total RNAs, fractionation of RNAs by formaldehyde agarose gel electrophoresis, RNA transfer to nylon membranes, and RNA blot hybridization have been described (Pautot et al. 1991). All gels were stained with ethidium bromide to assure equivalent loading per lane. All RNA blots were exposed to XAR5 film (Kodak, Rochester, N.Y., U.S.A.) at $-80^{\circ} \mathrm{C}$ with one Quanta III intensifying screen (Kodak). ${ }^{32} \mathrm{P}$-labeled LapAl-antisense RNAs were synthesized in vitro with T3 RNA polymerase (Gibco BRL, Cergy Pontoise, France) and $\left[\alpha-{ }^{32} \mathrm{P}\right]-\mathrm{CTP}$ (Amersham, Les Ulis, France) from the pBluescript-KS LapAl subclone. This subclone contained the 1.6-kb EcoRI-XbaI LapAl fragment from pDR57 (Pautot et al. 1993). DNA probes were labeled with $\left[\alpha-{ }^{32} \mathrm{P}\right]-\mathrm{dCTP}$ and the random priming method of Feinberg and Vogelstein (1983) or with a nick-translation kit (Gibco BRL). Under the stringent hybridization conditions used in these studies and described in Pautot et al. (1991), the ${ }^{32}$ P-labeled LapA probe detected LapA1 and LapA2 RNAs but did not cross hybridize with the constitutive, rare-class LapN transcripts (C.-J. Tu and L. Walling, unpublished results). The proteinase inhibitor II (pin2; pT2-47), $P R-1$ (pP6), and $P R-4$ (pPR-P2) cDNA clones have been described (Graham et al. 1985; Linthorst et al. 1991; Van Kan et al. 1992).

\section{Protein extraction, fractionation, immunoblots, and LAP activity assays.}

The procedures for the extraction of native, soluble proteins and aminopeptidase gel activity assays have been described
(Gu et al. 1996b). While the activities of the LAP-A and the fast migrating aminopeptidase (AMP1) were visualized in this assay, LAP-N activity was not detected. Swine LAP was purchased from Sigma (St. Louis, MO, U.S.A.) Total protein extraction, SDS-PAGE, 2D-PAGE, silver staining, and immunoblots were performed according to $\mathrm{Gu}$ et al. (1996b). A 1:500 dilution of the LAP-A polyclonal antisera was used in the immunoblot analyses. Immunoblots were performed with proteins from homozygous progeny of the 35S:asLapAl lines 3 and 4 plants.

\section{ACKNOWLEDGMENTS}

We thank D. Cooksey of the University of California, Riverside, for supplying the P. syringae pv. tomato strains; C. Bender of Oklahoma State University, Stillwater, for supplying coronatine; S. Jansen for providing Manduca sexta eggs; and T. Michael for providing the pLBR19 vector. We thank J.-M. Pollien for his aid in the insect feeding studies and C. J. Tu, W. S. Chao, and Y.-Q. Gu for helpful discussions. This work was supported by the INRA and NSF Grant IBN-9318260 to L. L. Walling.

\section{LITERATURE CITED}

An, G., Enert, P. R., Mitra, A., and Ha, S. B. 1988. Binary vectors. Pages 1-19 in: Plant Molecular Biology Manual. S. B. Gelvin and R. A. Schilperoot, eds. Kluwer Academic Publishers, Dordrecht, The Netherlands.

Bartling, D., and Weiler, E. W. 1992. Leucine aminopeptidase from Arabidopsis thaliana: Molecular evidence for a phylogenetically conserved enzyme of protein turnover in higher plants. Eur. J. Biochem. 205:425-431.

Bartling, D., and Nosek, J. 1994. Molecular and immunological characterization of leucine aminopeptidase in Arabidopsis thaliana: A new antibody suggests a semi-constitutive regulation of a phylogenetically old enzyme. Plant Sci. 99:199-209.

Bender, C. L., Stone, H. E., Sims, J. J., and Cooksey, D. A. 1987. Reduced pathogen fitness of Pseudomonas syringae pv. tomato Tn5 mutants in coronatine production. Physiol. Mol. Plant Pathol. 30:273283.

Bender, C. L., Rangaswamy, V., and Loper, J. 1999. Polyketide production by plant-associated pseudomonads. Annu. Rev. Phytopathol. 37:175-196.

Beninga, J., Rock, K. L., and Goldberg, A. L. 1998. Interferon- $\gamma$ can stimulate post-proteasomal trimming of the $\mathrm{N}$ terminus of an antigenic peptide by inducing leucine aminopeptidase. J. Biol. Chem. 273:18734-18742.

Bevan, M. 1984. Binary Agrobacterium vectors for plant transformation. Nucleic Acids Res. 12:8711-8721.

Cannon, M., Platz, J., Oleary, M., Sookdeo, C., and Cannon, F. 1990. Organ-specific modulation of gene expression in transgenic plants using antisense RNA. Plant Mol. Biol. 15:39-47.

Chao, W. S., Gu, Y.-Q., Pautot, V., Bray, E. A., and Walling, L. L. 1999. Leucine aminopeptidase RNAs, proteins and activities increase in response to water deficit, salinity and the wound signals-systemin, methyl jasmonate, and abscisic acid. Plant Physiol. 120:1-14.

Chao, W. S., Pautot, V., Holzer, F. M., and Walling, L. L. 2000. Leucine aminopeptidases: The ubiquity of LAP-N and the specificity of LAPA. Planta 210:563-573.

Dempsey, D. M. A., Shah, J., and Klessig, D. F. 1999. Salicylic acid and disease resistance in plants. Crit. Rev. Plant Sci. 18:547-575.

Doares, S. H., Narváez-Vásquez, J., Conconi, A., and Ryan, C. A. 1995. Salicylic acid inhibits synthesis of proteinase inhibitors in tomato leaves induced by systemin and jasmonic acid. Plant Physiol. 108:1741-1746.

Doherty, H. M., Sevendran, R. R., and Bowles, D. J. 1988. The wound response of tomato plants can be inhibited by aspirin and related hydroxy-benzoic acids. Physiol. Mol. Plant Pathol. 33:377-384.

Farmer, E. E., and Ryan, C. A. 1990. Interplant communication: Airborne methyl jasmonate induces synthesis of proteinase inhibitors in plant leaves. Proc. Natl. Acad. Sci. USA 87:7713-7716. 
Feinberg, A. P., and Vogelstein, B. 1983. A technique for radiolabeling DNA restriction endonuclease fragments to high specific activity. Anal. Biochem. 132:6-13.

Feys, B. J. F., Benedetti, C. E., Penfold, C. N., and Turner, J. G. 1994. Arabidopsis mutants selected for resistance to the phytotoxin coronatine are male sterile, insensitive to methyl jasmonate, and resistant to a bacterial pathogen. Plant Cell 6:751-759.

Graham, J. S., Pearce, G., Merryweather, J., Titani, K., Ericsson, L. H., and Ryan, C. A. 1985. Wound-induced proteinase inhibitors from tomato leaves. II. The cDNA-deduced primary structure of pre-inhibitor II. J. Biol. Chem. 260:6561-6564.

Gu, Y.-Q., and Walling, L. L. 2000. Specificity of the wound-induced leucine aminopeptidase (LAP-A) of tomato: Activity on dipeptide and tripeptide substrates. Eur. J. Biochem. 267:1178-1187.

Gu, Y. Q., Chao, W. S., and Walling, L. L. 1996a. Localization and posttranslational processing of the wound-induced leucine aminopeptidase proteins of tomato. J. Biol. Chem. 271:25880-25887.

Gu, Y. Q., Pautot, V., Holzer, F. M., and Walling, L. L. 1996b. A complex array of proteins related to the multimeric leucine aminopeptidase of tomato. Plant Physiol. 110:1257-1266.

Gu, Y.-Q., Holzer, F. M., and Walling, L. L. 1999. Over-expression, purification and biochemical characterization of the wound-induced leucine aminopeptidase of tomato. Eur. J. Biochem. 263:1-11.

Guerineau, F., Lucy, A., and Mullineaux, P. 1992. Effect of two consensus sequences preceding the translation initiator codon on gene expression in plant protoplasts. Plant Mol. Biol. 18:815-818.

Hamza, S., and Chupeau, Y. 1993. Re-evaluation of conditions for plant regeneration and Agrobacterium-mediated transformation from tomato (Lycopersicon esculentum). J. Exp. Bot. 44:1837-1845.

Harms, K., Atzorn, R., Brash, A., Kuehn, H., Wasternack, C., Willmitzer, L., and Peña-Cortés, H. 1995. Expression of a flax allene oxide synthase cDNA leads to increased endogenous jasmonic acid (JA) levels in transgenic potato plants but not to a corresponding activation of JA-responding genes. Plant Cell 7:1645-1654

Harris, C. A., Hunte, B., Krauss, M. R., Taylor, A., and Epstein, L. B. 1992. Induction of leucine aminopeptidase by interferon- $\gamma$ : Identification by protein microsequencing after purification by preparative 2dimensional gel electrophoresis. J. Biol. Chem. 267:6865-6869.

Herbers, K., Prat, S., and Willmitzer, L. 1994. Functional analysis of a leucine aminopeptidase from Solanum tuberosum L. Planta 194:230240.

Hildmann, T., Ebneth, M., Peña-Cortés, H., Sanchez Serrano, J. J., Willmitzer, L., and Prat, S. 1992. General roles of abscisic and jasmonic acids in gene activation as a result of mechanical wounding. Plant Cell 4:1157-1170.

Jia, Y. L., and Martin, G. B. 1999. Rapid transcript accumulation of pathogenesis-related genes during an incompatible interaction in bacterial speck disease-resistant tomato plants. Plant Mol. Biol. 40:455-465.

Kim, H., and Lipscomb, W. N. 1994. Structure and mechanism of bovine lens leucine aminopeptidase. Adv. Enzymol. Relat. Areas Mol. Biol. 68:153-213.

Koda, Y., Takahashi, K., Kikuta, Y., Greulich, F., Toshima, H., and Ichihara, A. 1996. Similarities of the biological activities of coronatine and coronafacic acid to those of jasmonic acid. Phytochemistry 41:93-96.

Linthorst, H. J. M., Danhash, N., Brederode, F. T., Van Kan, J. A. L., De Wit, P. J. G. M., and Bol, J. F. 1991. Tobacco and tomato PR proteins homologous to win and pro-hevein lack the "hevein" domain. Mol. Plant-Microbe Interact. 4:586-592.

Martin, G. B. 1999. Functional analysis of plant disease resistance genes and their downstream effectors. Curr. Opin. Plant Biol. 2:273-279.

Mikkonen, A. 1992. Purification and characterization of leucine aminopeptidase from kidney bean cotyledons. Physiol. Plant 84:393-398.

Milligan, S. B., and Gasser, C. S. 1995. Nature and regulation of pistilexpressed genes in tomato. Plant Mol. Biol. 28:691-711.

Mitchell, R. E., and Young, H. 1978. Identification of a chlorosisinducing toxin of Pseudomonas glycinea as coronatine. Phytochemistry 17:2028-2029.

Mittal, S., and Davis, K. R. 1995. Role of the phytotoxin coronatine in the infection of Arabidopsis thaliana by Pseudomonas syringae pv. tomato. Mol. Plant-Microbe Inter. 8:165-171.

Palmer, D. A., and Bender, C. L. 1995. Ultrastructure of tomato leaf tissue treated with the pseudomonad phytotoxin coronatine and com- parison with methyl jasmonate. Mol. Plant-Microbe Interact. 8:683692.

Pautot, V., Holzer, F. M., and Walling, L. L. 1991. Differential expression of tomato proteinase inhibitor I and inhibitor II genes during bacterial pathogen invasion and wounding. Mol. Plant-Microbe Interact. 4:284-292.

Pautot, V., Holzer, F. M., Reisch, B., and Walling, L. L. 1993. Leucine aminopeptidase: An inducible component of the defense response in Lycopersicon esculentum (tomato). Proc. Natl. Acad. Sci. USA 90:9906-9910.

Pearce, G., Strydom, D., Johnson, S., and Ryan, C. A. 1991. A polypeptide from tomato leaves induces wound-inducible proteinase inhibitor proteins. Science 253:895-898.

Peña-Cortés, H., Albrecht, T., Prat, S., Weiler, E. W., and Willmitzer, L. 1993. Aspirin prevents wound-induced gene expression in tomato leaves by blocking jasmonic acid biosynthesis. Planta 191:123-128.

Pieterse, C. M. J., and van Loon, L. C. 1999. Salicylic acid-independent plant defence pathways. Trends Plant Sci. 4:52-58.

Rawlings, N. D., and Barrett, A. J. 1993. Evolutionary families of peptidases. Biochem. J. 290:205-18.

Rojo, E., León, J., and Sánchez-Serrano, J. J. 1999. Cross-talk between wound signalling pathways determines local versus systemic gene expression in Arabidopsis thaliana. Plant J. 20:135-142.

Romeis, T., Piedras, P., Zhang, S., Klessig, D. F., Hirt, H., and Jones, J. D. G. 1999. Rapid Avr9- and Cf-9-dependent activation of MAP kinases in tobacco cell cultures and leaves: Convergence of resistance gene, elicitor, wound, and salicylate responses. Plant Cell 11:273-287.

Ruíz-Rivero, O. J., and Prat, S. 1998. A -308 deletion of the tomato LAP promoters is able to direct flower-specific and MeJA-induced expression in transgenic plants. Plant Mol. Biol. 36:639-648.

Ryan, C. A. 2000. The systemin signaling pathway: differential activation of defensive genes. Biochim. Biophys. Acta 1477:112-122.

Sano, H., Seo, S., Orudgev, E., Youssefian, S., Ishizuka, K., and Ohashi, Y. 1994. Expression of the gene for a small GTP binding protein in transgenic tobacco elevates endogenous cytokinin levels, abnormally induces salicylic acid in response to wounding, and increases resistance to tobacco mosaic virus infection. Proc. Natl. Acad. Sci. USA 91:10556-10560.

Schaller, A., and Ryan, C. A. 1996. Systemin: A polypeptide defense signal in plants. BioEssays 18:27-33.

Sembdner, G., and Parthier, B. 1993. The biochemistry and the physiological and molecular actions of jasmonates. Annu. Rev. Plant Physiol. Plant Mol. Biol. 44:569-589.

Seo, S., Okamoto, M., Seto, H., Ishizuka, K., Sano, H., and Ohashi, Y. 1995. Tobacco MAP kinase: a possible mediator in wound signal transduction pathways. Science 270:1988-1992.

Smith, E. L., and Hill, R. L. 1960. Leucine aminopeptidase. Pages 37-62 in: The Enzymes. P. D. Boyer, H. Lardy, and K. Myrback, eds. Academic Press, New York.

Sopanen, T., and Mikola, J. 1975. Purification and partial characterization of barley leucine aminopeptidase. Plant Physiol. 55:809-814.

Stockinger, E. J., and Walling, L. L. 1994. Pto3 and Pto4: Novel genes from Lycopersicon hirsutum var. glabratum that confer resistance to Pseudomonas syringae pv. tomato. Theor. Appl. Genet. 89:879-884.

Stuart, D. D., and Doughty, M. J. 1996. In vitro UVB irradiation of bovine crystalline lens causes cell damage and reduction in leucine aminopeptidase activity in lens epithelium. J. Photochem. Photobiol. B Biol. 32:81-7.

Taylor, A., Peltier, C. Z., Torre, F. J., and Hakamian, N. 1993. Inhibition of bovine lens leucine aminopeptidase by bestatin. Number of binding sites and slow binding of this inhibitor. Biochemistry 32:784-790.

Taylor, A., Sanford, D., and Nowell, T. 1996. Structure and function of bovine lens aminopeptidase and comparison with homologous aminopeptidases. Pages 21-67 in: Aminopeptidases. A. Taylor, ed. R. G. Landes Co., Austin, Texas.

van de Ven, W. T. G., LeVesque, C. S., Perring, T. M., and Walling, L. L. 2000. Local and systemic changes in squash gene expression in response to silverleaf whitefly feeding. Plant Cell 120:1409-1423.

van der Krol, A. R., Mur, L. A., Delange, P., Mol, J. N. M., and Stuitje, A. R. 1990. Inhibition of flower pigmentation by antisense $C H S$ genes: Promoter and minimal sequence requirements for the antisense effect. Plant Mol. Biol. 14:457-466.

van Kan, J. A. L., Joosten, M. H. A. J., Wagemakers, C. A. M., van den Berg-Velthuis, G. C. M., and de Wit, P. J. G. M. 1992. Differential ac- 
cumulation of messenger RNAs encoding extracellular and intracellular PR proteins in tomato induced by virulent and avirulent races of Cladosporium fulvum. Plant Mol. Biol. 20:513-527.

Walling, L. L. 2000. The myriad plant responses to herbivores. J. Plant Growth Regul. 19:195-216.

Walling, L. L., and Gu, Y.-Q. 1996. Plant aminopeptidases: Occurrence, function and characterization. Pages 174-219 in: Aminopeptidases. A Taylor, ed. R. G. Landes, Georgetown, Texas, U.S.A.
Wasternack, C., Ortel, B., Miersch, O., Kramell, R., Beale, M., Greulich, F., Feussner, I., Hause, B., Krumm, T., Boland, W., and Parthier, B. 1998. Diversity in octadecanoid-induced gene expression of tomato. J. Plant Physiol. 152:345-352.

Weiler, E. W., Kutchan, T. M., Gorba, T., Brodschelm, W., Niesel, U., and Bublitz, F. 1994. The Pseudomonas phytotoxin coronatine mimics octadecanoid signalling molecules of higher plants. FEBS Lett. 345:9-13. 